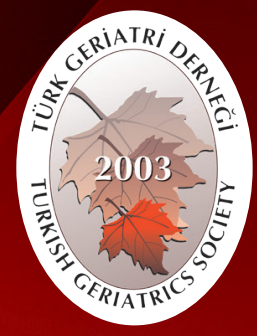

Turkish Journal of Geriatrics

DOI: 10.31086/tigeri.2019150579

2019;22 (1):101-111

- Hatice ŞiMŞEK ${ }^{1}$ (D)

- Hande BAHADIR ${ }^{1}$ D

- Ahmet Can BiLGiN² (D)

CORRESPONDANCE

Hatice ŞiMŞEK

Dokuz Eylul University, Faculty of Medicine

Department of Public Health, Izmir, Turkey

Phone: 02324124001

e-mail: haticesimsek@yahoo.com

Received: $27 / 04 / 2018$

Accepted: $31 / 01 / 2019$

Dokuz Eylul University Faculty of Medicine

Department of Public Health, İzmir, Turkey

Dokuz Eylul University, Faculty of Medicine,

Department of History of Medicine and Ethics, İmir, Turkey

\section{PHYSICIANS' ATTITUDES TOWARDS THE ELDERLY: AGEISM IN A UNIVERSITY HOSPITAL IN TURKEY}

\section{Abstract}

Introduction: The aim of this study was to determine attitudes of research assistant physicians towards the elderly in a university hospital and to evaluate the association of sociodemographic and occupational characteristics with ageism.

Materials and Methods: In this descriptive study, the target population comprised 521 research assistant physicians who worked the internal medicine and surgical clinics of the university hospital between December 2014 and March 2015. Variables considered in this study included demographic and occupational characteristics and attitudes towards the elderly. The Ageism Attitude Scale (AAS) was used to identify the physicians' attitudes towards the elderly. The data were collected using the sealed envelope system. Results are presented as percentage distribution and mean. A t-test was used for statistical analysis.

Results: A total of 327 physicians were included in this study. The total mean score obtained from the EDAS was $86.9 \pm 8.0$, with a score of $37.4 \pm 3.8$ for the Restricting the Life of the Elderly dimension, $30.2 \pm 4.3$ for the Positive Ageism dimension and 19.3 \pm 3.1 for the Negative Ageism dimension. No significant difference was observed between the mean attitude scores in terms of sociodemographic and occupational characteristics ( $p>0.05$ )

Conclusion: This study showed demonstrated that, in general, research assistant physicians exhibited a positive attitude towards the elderly.

Keywords: Aged; Attitude; Ageism

ARAŞTIRMA

\section{HEKIMLERDE YAŞLILARA ILIŞKIN TUTUMLAR: TÜRKIYE'DE BIR ÜNIVERSITE HASTANESINDE YAŞ AYRIMCILIĞI}

\section{$\ddot{O}_{z}$}

Giriş: Bir üniversite hastanesinde araştırma görevlisi olarak çalışan hekimlerin yaşlılara yönelik tutumlarını ve tutumlarının bazı sosyodemografik ve mesleki değişkenlere göre farklı olup olmadığını belirlemek amaçlandı.

Gereç ve Yöntem: Tanımlayıcı tipteki araştırmada İzmir'de bir üniversite hastanesinde Aralık 2014- Mart 2015 tarihleri arasında Dahili ve Cerrahi bilimlerde araştırma görevlisi olarak çalışan 521 hekime ulaşmak hedeflendi. Çalışmanın değişkenleri yaş ayrımcılığına ilişkin tutumun yanı sıra demografik ve mesleki özelliklerdi. Yaş ayrımcılığını belirlemek için Yaşlı Ayrımcılığı Tutum Ölçeği (YATÖ) kullanıldı. Veri kapalı zarf yöntemi ile toplandı, ortalama, yüzde dağılımları ile sunuldu t testi ile analiz edildi.

Bulgular: Çalışmada 327 hekimin verisi sunuldu. YATÖ'den alınan ortalama puanlar; toplamda $86.9 \pm 8.0$, yaşııın yaşamının sınırlanmasında $37.4 \pm 3.8$, pozitif ayrımcılıkta $30.2 \pm 4.3$, negatif ayrımcılıkta $19.3 \pm 3.1$ 'di. Demografik ve mesleki özelliklere göre YATÖ ortalama puanları arasında anlamlı fark bulunmadı ( $\mathrm{p}>0.05$ ).

Sonuç: Bir üniversite hastanesinde çalışan araştırma görevlisi hekimlerinin genelde, yaşlılara karşı olumlu tutum sergiledikleri belirlenmiştir.

Anahtar sözcükler: Yaşlı; Tutum; Yaş ayrımcılığı 


\section{INTRODUCTION}

The proportion of elderly individuals in the general population is gradually rising worldwide, including in Turkey. The population aged $\geq 65$ years in Turkey has increased by $17 \%$ in the last five years, and the elderly accounted for $8.3 \%$ of the entire population in 2016 (1). Such an increase in the proportion of elderly individuals corresponds with an increased need for healthcare and social support for the elderly. Ageism is defined as prejudice and discrimination based on a person's chronological age (2). Ageism increases needs for healthcare and social support for the elderly $(3,4)$ and diminishes access to and the quality of healthcare among elderly individuals (5-8). Conversely, as a result of their disease burden, elderly individuals encounter healthcare workers more frequently than young individuals, emphasizing the importance of the perceptions of healthcare personnel towards the elderly. Additionally, due to increasing elderly populations, physicians are required to provide healthcare services to an increasing number of elderly individuals each day. Healthcare problems among the elderly are more complex than those in young individuals. Additionally, negative attitudes of healthcare personnel may increase the complexity of health problems among the elderly (9).

Furthermore, the healthcare staff members, who are part of a system that favours early discharge, find it difficult to manage patients who experience longer disease duration and require more time to recuperate and rehabilitate. This characteristic of the healthcare system increases the impact of ageism (10).

Ageism studies have typically been conducted among students $(7,8,11,12)$. Moreover, studies regarding the attitudes of healthcare workers regarding ageism, particularly physicians, are scarce. Taken together, the abovementioned problems indicate the importance of determining the perceptions and attitudes of physicians towards the elderly.

Thus, the objectives of this study were to determine the perceptions and attitudes related to ageism of research assistant physicians working at the internal medicine and surgical clinics of the university hospital between December 2014 and March 2015 and to evaluate the association between sociodemographic and occupational characteristics and ageism.

\section{MATERIALS AND METHOD}

\section{Sample}

This descriptive study included 521 physicians working as research assistants at the internal medicine and surgical clinics of the university hospital between December 2014 and March 2015. All research assistant physicians during this time were included in this study without sampling.

\section{Variables}

Variables considered in this study included demographic and occupational characteristics and attitudes towards the elderly. The Ageism Attitude Scale (AAS) was used to identify the physicians' attitudes towards the elderly. The AAS is a 5-point Likert-type scale developed by Vefikuluçay, which comprises 25 items whose validity and reliability are confirmed by the choices 'Strongly Disagree', 'Disagree', 'Undecided', 'Agree' and 'Strongly Agree'. The Cronbach alfa reliability coefficient of this scale has been reported to be 0.80 . This scale includes both positive and negative attitude statements. Positive attitude statements are scored as follows: 5=Strongly Agree, 4=Agree, 3=Undecided, 2=Disagree and 1=Strongly Disagree. Negative attitude statements are scored in the opposite manner as that described above. The maximum and minimum obtainable points in the scale are '115' and '23', respectively. 
Positive attitude related to elder discrimination increases as the points in the scale increase. The scale comprises three dimensions as follows:

1. Restricting the Life of the Elderly: These are beliefs and perceptions of the society that are related to restricting the social lives of the elderly. The maximum and minimum obtainable scores in this dimension are '45' and '9', respectively.

2. Positive Ageism towards the Elderly: These are positive beliefs and perceptions of the society towards the elderly. The maximum and minimum obtainable scores in this dimension are '40' and '8', respectively.

3. Negative Ageism towards the Elderly: These are negative beliefs and perceptions of the society towards the elderly. The maximum and minimum obtainable scores in this dimension are '30' and '6', respectively (13).

\section{Data collection and analysis}

The questionnaires were distributed to the research assistant physicians and were collected by the sealed envelope system. Complementary results are presented as the percentage distribution and mean and standard deviation. The association between sociodemographic and occupational characteristics and ageism was analysed by t-test.

\section{Ethical issues}

This study was approved by Non-Invasive Research Ethics Committee of the university (2014/38-07).

\section{RESULTS}

A total of 327 physicians were included in this study (response rate, 62.8\%); 170 (54.7\%) of the participants were female, and the mean age of the participants was $30.4 \pm 3.0$ (range, 26-49) years. The mean total work experience was $6.2 \pm 2.9$ (range, 2-25) years, and the mean working time in a department was 24.4 \pm 16.6 (range, 1-96) months. The sociodemographic characteristics are presented in Table 1.

A total of $47.4 \% \quad(n=155)$ of the physicians had lived with an elderly individual during any period of their lives. Moreover, $75.5 \%(n=246)$ of the physicians stated that their family structure influenced their attitude towards the elderly, and $95.7 \%(n=313)$ of the physicians stated that departments that provide medical care for the elderly are necessary.

The total mean score obtained from the AAS was $86.9 \pm 8.0$ (range, 62-112), with a mean score of $37.4 \pm 3.8$ (range, 27-45) for the Restricting the Life of the Elderly dimension, 30.2 \pm 4.3 (range, 14-40) for the Positive Ageism dimension and 19.3 \pm 3.1 (range, 11-28) for the Negative Ageism dimension.

The lowest mean score in the Restricting the Life of the Elderly dimension of the AAS $(n=3.7 \pm 0.9)$ was observed in the item 'Elderly people can't carry bags and packages without help' (Table 2). The lowest mean score in the Positive Ageism dimension of the AAS was observed in the items 'the elderly are more likely to be patient than young people' $(3.0 \pm 1.1)$, 'the elderly are more tolerant than young people' (3.2 \pm 0.9$)$ and 'the elderly are more compassionate' $(3.7 \pm 0.8)$ (Table 3). The lowest mean score in the Negative Ageism dimension of the AAS was observed in the items 'preference should be given to young people over the elderly' (2.8 \pm 1.0$)$ and 'the elderly are not able

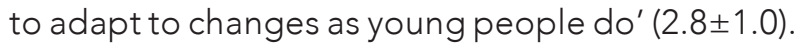
Generally, the Negative Ageism dimension was the dimension with the lowest mean scores (Table 3).

No significant difference was observed between the mean attitude scores in terms of sociodemographic and occupational characteristics ( $p>0.005)$ (Table 4). 
Table 1. Sociodemographic characteristics of the study population.

\begin{tabular}{|c|c|}
\hline Characteristics & n (\%) \\
\hline \multicolumn{2}{|c|}{ Age group (years; $n=327$ ) } \\
\hline 20-29 & $145(44.3)$ \\
\hline 30-39 & $179(54.7)$ \\
\hline$>40$ & $3(0.9)$ \\
\hline \multicolumn{2}{|l|}{ Marital status $(n=327)$} \\
\hline Married & $134(41.0)$ \\
\hline Single & $184(56.3)$ \\
\hline Widowed or divorced & $9(2.8)$ \\
\hline \multicolumn{2}{|l|}{ Family type $(n=326)$} \\
\hline Nuclear family & $298(91.4)$ \\
\hline Extended family & $28(8.6)$ \\
\hline \multicolumn{2}{|c|}{ Place where participant resided the longest $(n=327)$} \\
\hline Rural area & $24(7.3)$ \\
\hline Urban area & $303(92.7)$ \\
\hline \multicolumn{2}{|c|}{ Department (clinic) where employed $(n=327)$} \\
\hline Internal Medicine & $257(78.6)$ \\
\hline Surgery & $70(21.4)$ \\
\hline \multicolumn{2}{|c|}{ Total work experience (years; $n=327$ ) } \\
\hline $1-9$ & $291(89.0)$ \\
\hline$>10$ & $36(11.0)$ \\
\hline \multicolumn{2}{|c|}{ Working time in a department (months; $n=326$ ) } \\
\hline $0-11$ & $80(24.5)$ \\
\hline $12-23$ & $82(25.2)$ \\
\hline 24-35 & $63(19.3)$ \\
\hline$>36$ & $101(31.0)$ \\
\hline \multicolumn{2}{|c|}{ Work-related satisfaction $(n=327)$} \\
\hline Satisfied & $263(80.4)$ \\
\hline Dissatisfied & $64(19.6)$ \\
\hline
\end{tabular}


Table 2. Descriptive statistics for the 'Restricting the Life of the Elderly' dimension.

\begin{tabular}{|c|c|c|c|c|c|c|}
\hline $\begin{array}{l}\text { Restricting the Life of the } \\
\text { Elderly }\end{array}$ & $\begin{array}{r}\text { Strongly } \\
\text { Disagree } \\
\mathrm{n}(\%)\end{array}$ & $\begin{array}{r}\text { Disagree } \\
\mathrm{n}(\%)\end{array}$ & $\begin{array}{r}\text { Undecided } \\
\text { n (\%) }\end{array}$ & $\begin{array}{r}\text { Agree } \\
\text { n (\%) }\end{array}$ & $\begin{array}{r}\text { Strongly } \\
\text { Agree } \\
\mathrm{n}(\%)\end{array}$ & mean $\pm s d$ \\
\hline $\begin{array}{l}\text { Lives of the elderly should be } \\
\text { limited to their homes }\end{array}$ & $196(59.9)$ & $115(35.2)$ & $11(3.4)$ & $2(0.6)$ & $3(0.9)$ & $4.5 \pm 0.7$ \\
\hline $\begin{array}{l}\text { The external appearance of the } \\
\text { elderly is repulsive }\end{array}$ & $158(48.3)$ & $128(39.1)$ & $18(5.5)$ & $9(2.8)$ & $14(4.3)$ & $4.2 \pm 1.0$ \\
\hline $\begin{array}{l}\text { It is unnecessary for the elderly } \\
\text { to buy homes, cars, possessions } \\
\text { or clothes }\end{array}$ & 137 (41.9) & 157 (48.0) & $22(6.7)$ & $11(3.4)$ & $0(0.0)$ & $4.3 \pm 0.7$ \\
\hline $\begin{array}{l}\text { Elderly people who lose their } \\
\text { spouses should not remarry }\end{array}$ & $119(36.4)$ & $134(41.0)$ & $61(18.7)$ & $12(3.7)$ & $1(0.3)$ & $4.1 \pm 0.8$ \\
\hline $\begin{array}{l}\text { The elderly should live in homes } \\
\text { for the elderly }\end{array}$ & $101(30.9)$ & $133(40.7)$ & 77 (23.5) & $14(4.3)$ & $2(0.6)$ & $4.0 \pm 0.9$ \\
\hline $\begin{array}{l}\text { Preference should be given to } \\
\text { the care of young people over } \\
\text { that of the elderly in a hospital } \\
\text { setting }\end{array}$ & 115 (35.2) & $166(50.8)$ & 29 (8.9) & $14(4.3)$ & $3(0.9)$ & $4.2 \pm 0.8$ \\
\hline $\begin{array}{l}\text { Elderly people should be paid } \\
\text { less than young people in the } \\
\text { work force }\end{array}$ & $134(41.0)$ & 155 (47.4) & $28(8.6)$ & $6(1.8)$ & $4(1.2)$ & $4.3 \pm 0.8$ \\
\hline $\begin{array}{l}\text { Elderly people cannot carry } \\
\text { bags and packages without help }\end{array}$ & $41(12.5)$ & $172(52.6)$ & $83(25.4)$ & $28(8.6)$ & $3(0.9)$ & $3.7 \pm 0.8$ \\
\hline $\begin{array}{l}\text { The care of the elderly should } \\
\text { not be considered an economic } \\
\text { burden by family members }\end{array}$ & $10(3.1)$ & $7(2.1)$ & $18(5.5)$ & $\begin{array}{r}153 \\
(46.8)\end{array}$ & $139(42.5)$ & $4.2 \pm 0.9$ \\
\hline
\end{tabular}


Table 3. Descriptive statistics of the 'Positive Ageism' and 'Negative Ageism' dimension.

\begin{tabular}{|c|c|c|c|c|c|c|}
\hline Dimension & $\begin{array}{r}\text { Strongly } \\
\text { Disagree } \\
\mathrm{n}(\%)\end{array}$ & $\begin{array}{r}\text { Disagree } \\
\mathrm{n}(\%)\end{array}$ & $\begin{array}{r}\text { Undecided } \\
\text { n (\%) }\end{array}$ & $\begin{array}{l}\text { Agree } \\
\text { n (\%) }\end{array}$ & $\begin{array}{r}\text { Strongly } \\
\text { Agree } \\
\mathrm{n}(\%)\end{array}$ & mean \pm sd \\
\hline \multicolumn{7}{|l|}{ Positive Ageism } \\
\hline $\begin{array}{l}\text { Elderly people are more patient than } \\
\text { young people }\end{array}$ & $24(7.3)$ & $95(29.1)$ & $84(25.7)$ & $\begin{array}{r}103 \\
(31.5)\end{array}$ & $21(6.4)$ & $3.0 \pm 1.1$ \\
\hline $\begin{array}{l}\text { Preference should be given to the } \\
\text { elderly in places where waiting in line } \\
\text { is required }\end{array}$ & $9(2.8)$ & $8(2.4)$ & $25(7.6)$ & $\begin{array}{r}130 \\
(39.8)\end{array}$ & $155(47.4)$ & $4.3 \pm 0.9$ \\
\hline $\begin{array}{l}\text { Young people should learn from the } \\
\text { experiences of elderly people }\end{array}$ & $5(1.5)$ & $10(3.1)$ & $47(14.4)$ & $\begin{array}{r}163 \\
(49.8)\end{array}$ & $102(31.2)$ & $4.1 \pm 0.9$ \\
\hline $\begin{array}{l}\text { The elderly should be shown } \\
\text { importance by the family members } \\
\text { with whom they live }\end{array}$ & $7(2.1)$ & $4(1.2)$ & $5(1.5)$ & $\begin{array}{r}148 \\
(45.3)\end{array}$ & $163(49.8)$ & $4.4 \pm 0.8$ \\
\hline The elderly are more compassionate & $3(0.9)$ & $14(4.3)$ & $104(31.8)$ & $\begin{array}{r}153 \\
(46.8)\end{array}$ & $53(16.2)$ & $3.7 \pm 0.8$ \\
\hline $\begin{array}{l}\text { When decisions are made in the family, } \\
\text { the opinions of the elderly should be } \\
\text { considered }\end{array}$ & $6(1.8)$ & $10(3.1)$ & $72(22.0)$ & $\begin{array}{r}192 \\
(58.7)\end{array}$ & $47(14.4)$ & $3.8 \pm 0.8$ \\
\hline $\begin{array}{l}\text { The elderly are more tolerant than } \\
\text { young people }\end{array}$ & $10(3.1)$ & $63(19.3)$ & $136(41.6)$ & $99(30.3)$ & $19(5.8)$ & $3.2 \pm 0.9$ \\
\hline $\begin{array}{l}\text { When the family budget is being } \\
\text { developed, the opinions of the elderly } \\
\text { should be sought }\end{array}$ & $4(1.2)$ & $24(7.3)$ & $56(17.1)$ & $\begin{array}{r}208 \\
(63.6)\end{array}$ & $35(10.7)$ & $3.8 \pm 0.8$ \\
\hline \multicolumn{7}{|l|}{ Negative Ageism } \\
\hline Elderly people are always ill & $14(4.3)$ & $151(46.2)$ & $66(20.2)$ & $85(26.0)$ & $11(3.4)$ & $3.2 \pm 1.0$ \\
\hline $\begin{array}{l}\text { The basic responsibility of the elderly } \\
\text { should be to help their children } \\
\text { with tasks, such as housework, } \\
\text { kitchen chores and the care of their } \\
\text { grandchildren }\end{array}$ & $59(18.0)$ & $185(56.6)$ & $48(14.7)$ & $31(9.5)$ & $4(1.2)$ & $3.8 \pm 0.9$ \\
\hline $\begin{array}{l}\text { Preference should be given to young } \\
\text { people over the elderly when they are } \\
\text { hired for jobs }\end{array}$ & $21(6.4)$ & $52(15.9)$ & $116(35.5)$ & $\begin{array}{r}113 \\
(34.6)\end{array}$ & $25(7.6)$ & $2.8 \pm 1.0$ \\
\hline $\begin{array}{l}\text { Elderly people are not able to adapt to } \\
\text { changes like young people }\end{array}$ & $20(6.0)$ & $55(16.6)$ & $96(29.4)$ & $\begin{array}{r}143 \\
(43.7) \\
\end{array}$ & $15(4.6)$ & $2.8 \pm 1.0$ \\
\hline $\begin{array}{l}\text { Preference should be given to } \\
\text { young people for promotions } \\
\text { in work situations }\end{array}$ & $17(5.2)$ & $89(27.2)$ & $105(32.1)$ & $95(29.1)$ & $21(6.4)$ & $3.0 \pm 1.0$ \\
\hline $\begin{array}{l}\text { Elderly people should not go outside } \\
\text { on their own }\end{array}$ & $55(16.8)$ & $176(53.8)$ & $61(18.7)$ & $32(9.8)$ & $3(0.9)$ & $3.8 \pm 0.9$ \\
\hline
\end{tabular}




\begin{tabular}{|c|c|c|c|c|c|c|c|c|c|c|c|c|c|c|c|c|c|c|c|c|}
\hline a & 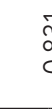 & & & & 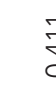 & & 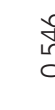 & & 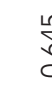 & & & 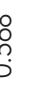 & 㘶 & & 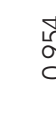 & & & ס. & & $\frac{j}{j}$ \\
\hline 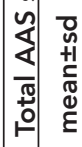 & $\begin{array}{l}\sim \\
\infty \\
+1 \\
\vdots \\
\vdots \\
\infty \\
\infty\end{array}$ & $\begin{array}{l}0 \\
\infty \\
0 \\
+1 \\
0 \\
0 \\
\infty \\
\infty\end{array}$ & 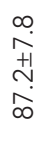 & $\begin{array}{l}0 \\
\infty \\
+1 \\
0 \\
0 \\
\infty \\
\infty\end{array}$ & 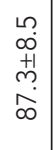 & 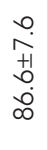 & $\begin{array}{l}\infty \\
\uparrow \\
+1 \\
\infty \\
0 \\
\infty \\
\infty\end{array}$ & $\begin{array}{c}\stackrel{N}{0} \\
\stackrel{0}{0} \\
+1 \\
\infty \\
\infty \\
\infty\end{array}$ & 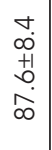 & $\begin{array}{l}0 \\
\infty \\
+1 \\
0 \\
0 \\
\infty\end{array}$ & $\begin{array}{c}n \\
\infty \\
+ \\
0 \\
\stackrel{\infty}{\infty} \\
\infty\end{array}$ & 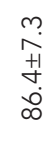 & 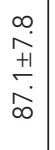 & $\begin{array}{l}\stackrel{ }{\infty} \\
\stackrel{0}{+} \\
\stackrel{+}{0} \\
\infty\end{array}$ & $\begin{array}{l}0 \\
\infty \\
+1 \\
0 \\
0 \\
\infty\end{array}$ & $\begin{array}{c}m \\
\infty \\
+ \\
0 \\
\stackrel{1}{0} \\
\infty\end{array}$ & $\begin{array}{c}N \\
\infty \\
+1 \\
0 \\
\infty \\
\infty\end{array}$ & 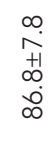 & $\begin{array}{c}N \\
o \\
+1 \\
m \\
\infty \\
\infty \\
\infty\end{array}$ & $\begin{array}{l}\infty \\
\stackrel{\infty}{+} \\
\text { th } \\
\stackrel{0}{\infty} \\
\infty\end{array}$ \\
\hline
\end{tabular}

\begin{tabular}{|c|c|c|c|c|c|c|c|c|c|c|c|c|c|c|c|c|c|c|c|c|}
\hline & & $\alpha$ & & $\begin{array}{l}\text { of } \\
\text { to }\end{array}$ & & & & & & & cે & & $\begin{array}{l}\text { on } \\
\text { a }\end{array}$ & & & & ơ & & סू & \\
\hline 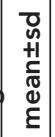 & 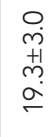 & 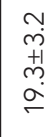 & 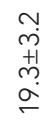 & 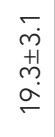 & $\begin{array}{l}\hat{m} \\
\dot{p} \\
\dot{1} \\
\tilde{a} \\
\underline{\sigma}\end{array}$ & 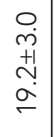 & 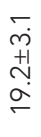 & 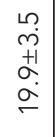 & 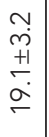 & $\begin{array}{c}\bar{m} \\
\vec{p} \\
m \\
\tilde{m} \\
\dot{\sigma}\end{array}$ & 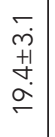 & $\begin{array}{l}\bar{r} \\
\vec{p} \\
\stackrel{0}{0} \\
\stackrel{a}{-}\end{array}$ & 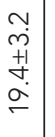 & $\begin{array}{l}0 \\
0 \\
+1 \\
0 \\
\infty \\
\infty\end{array}$ & 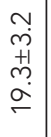 & $\begin{array}{l}0 \\
\dot{p} \\
+1 \\
\frac{1}{\sigma} \\
\sigma\end{array}$ & $\begin{array}{l}\underset{\sim}{m} \\
\stackrel{+}{\sim} \\
\stackrel{\sigma}{-}\end{array}$ & 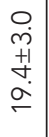 & 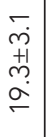 & 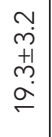 \\
\hline
\end{tabular}

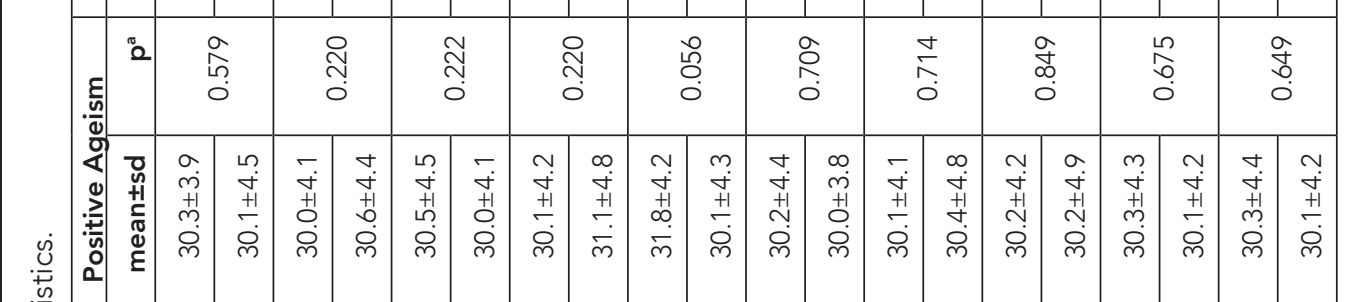

\begin{tabular}{|c|c|c|c|c|c|c|c|c|c|c|c|c|c|c|c|c|c|c|c|}
\hline 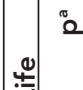 & 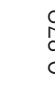 & 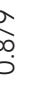 & a & & $\begin{array}{l}\mathbb{J} \\
\infty \\
0\end{array}$ & & 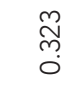 & & 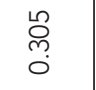 & శ్ & & 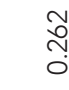 & & $\begin{array}{l}\stackrel{\overbrace{}}{\sim} \\
\stackrel{0}{0} \\
0\end{array}$ & & 남 & R & & \\
\hline 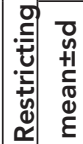 & 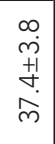 & 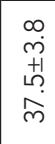 & 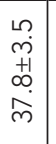 & $\begin{array}{l}a \\
\dot{m} \\
+ \\
0 \\
\dot{m} \\
\dot{m}\end{array}$ & 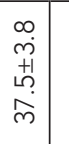 & 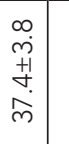 & 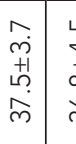 & 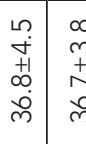 & 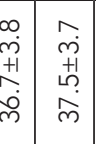 & $\begin{array}{l}\infty \\
m \\
+ \\
+ \\
\dot{m}\end{array}$ & $\begin{array}{l}\stackrel{a}{m} \\
\stackrel{m}{+} \\
\stackrel{\dot{m}}{m}\end{array}$ & $\begin{array}{l}\infty \\
m \\
\stackrel{1}{n} \\
\stackrel{1}{p} \\
m\end{array}$ & 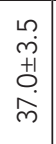 & $\begin{array}{l}\hat{m} \\
\dot{m} \\
+ \\
\dot{m} \\
\dot{m}\end{array}$ & $\begin{array}{l}0 \\
+ \\
+ \\
+1 \\
\infty \\
\tilde{m} \\
\tilde{m}\end{array}$ & $\begin{array}{l}\hat{m} \\
+ \\
+ \\
\stackrel{m}{m}\end{array}$ & 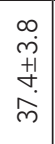 & $\begin{array}{l}\stackrel{0}{+} \\
\dot{+} \\
\stackrel{1}{1} \\
\hat{m}\end{array}$ & $\begin{array}{l}\stackrel{\sim}{\oplus} \\
\stackrel{+}{\stackrel{1}{*}} \\
\stackrel{m}{m}\end{array}$ \\
\hline \multirow[b]{2}{*}{ 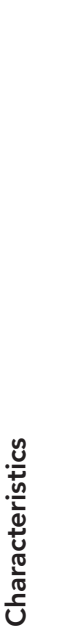 } & 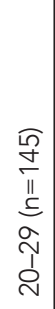 & 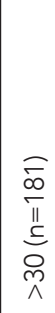 & 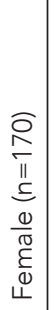 & 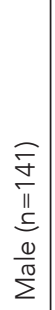 & 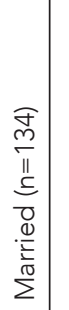 & 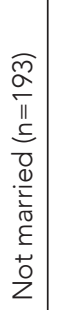 & 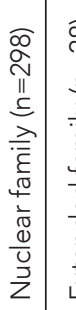 & 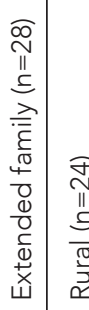 & 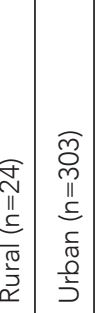 & 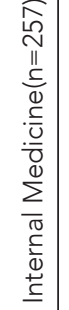 & 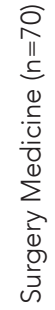 & 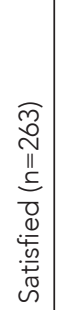 & 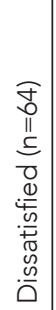 & 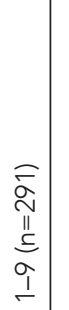 & 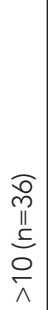 & 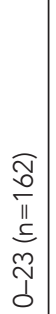 & 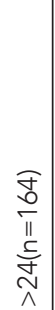 & 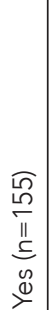 & $\begin{array}{l}\underset{N}{N} \\
\underset{N}{\| 1} \\
\underline{0} \\
0 \\
z\end{array}$ \\
\hline & \multicolumn{2}{|c|}{ 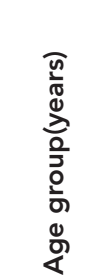 } & \multicolumn{2}{|c|}{ 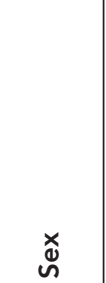 } & \multicolumn{2}{|c|}{ 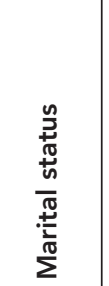 } & \multicolumn{2}{|l|}{ 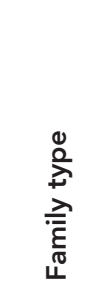 } & 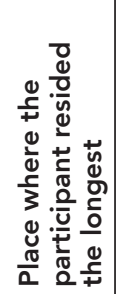 & \multicolumn{2}{|c|}{ 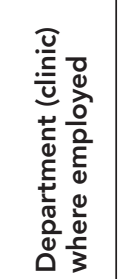 } & \multicolumn{2}{|c|}{ 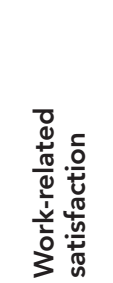 } & \multicolumn{2}{|c|}{ 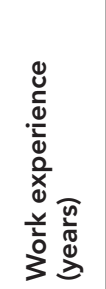 } & \multicolumn{2}{|c|}{ 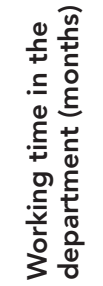 } & \multicolumn{2}{|c|}{ 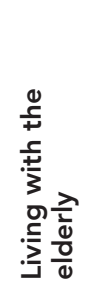 } \\
\hline
\end{tabular}




\section{DISCUSSION}

In this study, the attitudes on ageism of the research assistant physicians who worked at the internal medicine and surgical clinics of the university hospital were determined. Our findings revealed that, in general, physicians expressed a positive attitude regarding the elderly (mean score on AAS, 86.9). Additionally, the mean scores were 37.4 for the 'Restricting the Life of the Elderly' dimension, 30.2 in the Positive Ageism dimension and 19.3 in the Negative Ageism dimension. The study did not set a cut-off score, a value above which would have indicated a negative attitude, while a value below which would have indicated a positive attitude. However, two studies performed in a group of physicians in Turkey have reported similar scores (range, 83.1-86.6) (14,15), while only one study has revealed a lower score (68.4) (16). This score was assessed as a 'positive attitude' by the investigators who performed the study. The mean scores obtained in those studies using the same scale were as follows: 80.0 among nurses (14), 84.0-87.0 among students at a nursing school $(17,18), 83.7$ among the students of the Faculty of Economics and Administrative Sciences (19) and 70.6 among the students of the Vocational School of Health Services (20). The score obtained in a study that was performed in the general population was 80.2 (21). Generally, such high mean scores are obtained due to certain aspects of Turkish culture, such as traditional and unchanging expectations of respect and obedience towards the elderly and the protection of the elderly. Additionally, in our study, three out of every four physicians believed that family structure would influence their attitude towards the elderly, which demonstrates the importance of cultural influence. However, the social status of the elderly in Turkish culture varies, particularly in metropolitan areas. The reasons for this variation could be explained by increases in urbanisation, migration and industrialisation, economic difficulties, women's participation in the workforce, changes in individuals' social lives and changes in family structures (22). Nevertheless, this variation should be taken into consideration.

Other studies that used different scales have shown that attitudes towards the elderly and older patients range from neutral to positive among physicians (23-25). Some studies have also provided the evidence of the existence of ageism $(26,27)$.

In this study, although the total mean score was high, negative attitudes were also discovered when the scores for individual items were assessed. The lowest mean score in the Restricting the Life of the Elderly dimension of the AAS was observed in the item 'Elderly people cannot carry bags and packages without help', indicating that the elderly are considered to be weak or helpless. The lowest mean score in the Positive Ageism dimension of the AAS was observed in the items 'the elderly are more patient than young people', 'the elderly are more tolerant than young people' and 'the elderly are more compassionate'. The highest scores for the Positive Ageism dimension were observed in the items 'Priority should be given to the elderly in places where waiting in line is required' and 'The elderly should be shown importance by the family members with whom they live'. This positive attitude is driven by the society's expectation of respect for the elderly, which is embedded in their culture. Nonetheless, negative attitudes towards the individual characteristics of the elderly can also be observed.

In the present study, compared with other dimensions, the mean scores of the items in the Negative Ageism dimension were the lowest. The Negative Ageism dimension revealed that physicians who are research assistants believed that young people should be given priority over the elderly for recruitment and promotions. This attitude may be due to high levels of unemployment and the competitive nature of professional life. 
In this study, no significant difference was observed between the mean attitude scores of physicians according to age group, sex, marital status, family type, the location where the physician has lived the longest, department (clinic) where employed, work-related satisfaction, work experience, working time in the department or whether they had lived with the elderly. No significant association was found between ageism and sex in the study conducted by Kearney et al. (27), and no significant associations of ageism were found with sex, marital status, birth place and family structure in the study conducted by Ögenler et al. (15). Similarly, no significant associations of ageism were observed with sex, marital status, family structure and working hours in the study performed by Ünalan et al. (16) as well as with age, marital status, living arrangement and years of practice since graduation in the study conducted by Lui (24). Finally, no significant associations of ageism were reported with age, sex and years of practice in the study performed by Polat et al. (14). In contrast, the study performed by Leung et al. has revealed that physicians' characteristics that are associated with more positive attitudes towards the elderly included age of $\geq 30$ years, female sex and postgraduate years of $\geq 10$ (23). In the study performed by Ünalan et al., participants who had previously lived with an elderly family member exhibited a positive attitude towards elderly people (16). All of these studies were performed in a population of physicians and other healthcare workers. However, these different results may be due to differences between the scales used as well as cultural differences. The study performed by Elbi et al. is an important study as it demonstrates how the scale used can affect the results obtained. In their study, the association between sex and attitude revealed different results using different scales (28).

This study has some limitations. This study was performed only on physicians in a university hospital setting in a specific region of Turkey. In addition, since the sample size was small, the generalizability of the findings is limited. The overall response rate was moderate. Low participation could have been due to the lack of interest in the subject matter and lack of free time. Individuals who have participated in the study may have an interest in geriatrics and may already have a more positive attitude towards the elderly. Additionally, in studies in which an attitude is determined, the social desirability response bias should also be considered. Further, how different attitudes are reflected in behaviour remains unknown. Nevertheless, this study is important since it is one of the rare studies performed on resident physicians at a university hospital. Previous studies performed in Turkey and in other countries were typically performed on students.

In conclusion, physicians generally demonstrate positive attitudes towards the elderly. Nonetheless, when the individual items of the scale are reviewed, some negative attitudes may be observed. The physicians' attitudes towards the elderly are not related to their sociodemographic characteristics or working conditions. Although positive attitudes towards the elderly are considered to be influenced by cultural structure, it should be considered that the cultural structure changes over time. The establishment of theoretical and practical training that will encourage positive attitudes towards the elderly is imperative in medical education. For instance, contact with healthy elderly individuals during the early stages of education may lead to an improvement in attitude (23). Furthermore, considering the elderly patients who require healthcare services, the development of the concept of 'elder-friendly' would prevent negative attitudes or at least reduce their effects. Thus, studies that investigate these types of interventions on the attitudes of physicians towards the elderly are warranted.

\section{ACKNOWLEDGEMENTS}

We would like to thank Isik Top, Ozge Aksoy and Ozden Ozillice for assistance in data collection. 


\section{REFERENCES}

1. Turkish Statistical Institute. Older People in Statistics, 2016. News Bulletin Number: 24644, 16 Mart 2017. (Internet) Available from: http:// www.tuik.gov.tr/PreHaberBultenleri.do?id=24644 Accessed:19.01.2018. (in Turkish).

2. Butler RN. Age-ism: another form of bigotry. Gerontologist 1969;9(4):243-6. (PMID:5366225).

3. Lewis T, Barnes LL, Bienas JL, Lackland DT, Evans DA, Mendes de Leon CF. Perceived discrimination and blood pressure in older African Americans and white adults. J Gerontol A Biol Sci Med Sci 2009 Sep;64(9):1002-8. (PMID:19429703).

4. Westerhof GJ, Barrett AE. Age identity and subjective well-being: a comparison of the United States and Germany. J Gerontol B Psychol Sci Soc Sci 2005 May;60(3):129-36. (PMID:15860789).

5. Nelson TD. Ageism: prejudice against our feared future self. J Soc Issues 2005;61/2:207-21.

6. Clark A. Ageism and age discrimination in social care in the United Kingdom. Centre for Policy on Ageing, 2009, pp 8-12. (Internet) Available from: http://www. cpa.org.uk/information/reviews/CPA-\%20ageism and_age_discrimination_in_social_care-report.pdf. Accessed:17.02.2018.

7. Fabiano JA, Waldrop DP, Nochajski TH, Davis EL, Goldberg LJ. Understanding dental students' knowledge and perceptions of older people: toward a new model of geriatric dental education. J Dent Educ 2005;69:419-33. (PMID:15800255).

8. Kishimoto M, Nagoshi M, Williams S, Masaki K, Lanoie-Blanchette P. Knowledge and attitudes about geriatrics of medical students, internal medical residents and geriatric medicine fellows. J Am Ger Soc 2005;53:99-102. (PMID:15667384).

9. Deasey $D$, Kable A, Jeong S. Influence of nurses' knowledge of ageing and attitudes towards older people on therapeutic interactions in emergency care: A literature review. Australas. J Ageing 2014;33(4):229-36. (PMID:25471745).

10. Kydd A, Fleming A. Ageism and age discrimination in health care: fact or fiction? A narrative review of the literature. Maturitas. 2015 Aug;81(4):432-8. (PMID:26044073).

11. Ayoglu FN, Kulakci H, Ayyildiz TK, Aslan GK, Veren F. Attitudes of Turkish nursing and medical students toward elderly people. J Transcult Nurs 2013;25(3):241-8. (PMID:24381117).
12. Liu $Y$, While AE, Norman IJ, Ye W. Health professionals' attitudes toward older people and older patients: a systematic review. J Interprof Care 2012 Sep;26(5):397-409. (PMID:22780579).

13. Vefikulucay Yilmaz D, Terzioglu F. Development and psychometric evaluation of ageism attitude scale among the university students. Turkish Journal of Geriatrics 2011;14(3):259-68.

14. Polat U, Karadag Ayise, Ulger Z, Demir N. Nurses' and physicians' perceptions of older people and attitudes towards older people: Ageism in a hospital in Turkey. Contemp Nurse 2014;48(1):88-97. (PMID:25410199).

15. Ogenler O, Yapici G, Tasdelen B, Akca T. Opinions of a group medical doctors on elder discrimination in Mersin. Turkish Journal of Geriatrics 2012;15(4):40915. (in Turkish).

16. Unalan D, Soyuer F, Elmali F. Evaluation of the attitudes of nursing students towards ageism. Kafkas J Med Sci 2012;2(3):115-20. (in Turkish).

17. Yildirim Usta $Y$, Demir $Y$, Yonder M, Yildiz A. Nursing students' attitudes toward ageism in Turkey. Arch Gerontol Geriatr 2012;54:90-3. (PMID:21353316).

18. Ozdemir $O$, Bilgili N. Attitudes of Turkish nursing students related to ageism. J Nurs Res 2016 Sep;24(3):211-6. (PMID:26998768).

19. Sahin H. Research on university students' attitudes about elders and elder discrimination. Turkish Journal of Geriatrics 2015;18(1):47-53.

20. Soyuer F, Unalan D, Guleser N, Elmali F. The attitudes of Health Vocational School towards ageism and the relation of these ttitudes with some demographical variables. Mersin Univ Saglik Bilim Derg 2010;3(2):2025. (in Turkish).

21. Yilmaz $M$, Altiok $M$, Polat $B$, Darici $M$, Sungur MA. Attitudes of young adults toward ageism. Turkish Journal of Geriatrics 2012;15(4):416-23. (in Turkish).

22. Cilingiroglu N, Demirel S. Aging and ageism. Turkish Journal of Geriatrics 2004;7(4):225-30. (in Turkish).

23. Leung S, Logiudice D, Schwarz J, Brand C. Hospital doctors' attitudes towards older people. Intern Med J. 2011 Apr;41(4):308-14. (PMID:20002850)

24. Lui NL, Wong $\mathrm{CH}$. Junior doctors' attitudes towards older adults and its correlates in a tertiary-care public hospital. Ann Acad Med Singapore 2009;38(2):125-9. (PMID:19271039). 
25. Gunderson A, Tomkowiak J, Menachemi N, Brooks R. Rural physicians' attitudes toward the elderly: Evidence of ageism? Qual Manag Health Care 2005;14(3):167-76. (PMID:16027595).

26. Dobrowolska B, Jędrzejkiewicz B, Pilewska-Kozak A, et al. Age discrimination in healthcare institutions perceived by seniors and students. Nurs Ethics 2017 Jan 1:969733017718392. (PMID:28745574).
27. Kearney N, Miller M, Paul J, Smith K. Oncology healthcare professionals' attitudes toward elderly people. Ann Oncol 2000;11(5):599-601. (PMID:10907955).

28. Elbi H, Altan S, Rahman S, Cengiz Ozyurt B, Sahin S, Cam FS. The attitudes of medical students toward elderly. Turkish Journal of Geriatrics 2015;18(4):299304. 